\title{
Progress and Problems in the Prescribing/Dispensing Split and “Divided Package Sales" by Wholesalers
}

\author{
Makoto ShIRAGami, ${ }^{*}, 1$ Mitsuo MoriKubo, ${ }^{2}$ Ryuta SUZUKI, ${ }^{2}$ and Ichiro KUdo ${ }^{1}$ \\ Department of Health Chemicals, School of Pharmaceutical Science, Showa University, ${ }^{1}$ \\ 1-5-8, Hatanodai, Shinagawa-ku, Tokyo, 142-8555, Japan and Toho Yakuhin \\ Co. Ltd., ${ }^{2}$ 5-2-1, Daizawa, Setagaya-ku, Tokyo 155-8655, Japan
}

(Received March 27, 2000; Accepted September 12, 2000)

\begin{abstract}
Given the rapid progress of the prescribing/dispensing split, the ability of pharmacies to obtain and stock a small quantity of pharmaceuticals is seen as essential. Toho Yakuhin's experience in "divided package sales" (wholesalers open an original package supplied by the manufacturer and sell only a part of the contents) shows that the number of orders and sales amount, and the number of pharmacies ordering in sales have remarkably increased. One or two kinds of pharmaceuticals were ordered in $73 \%$ cases, and one or two units in about $50 \%$. This implies that "divided package sales" are utilized to obtain rarely prescribed drugs. On the other hand, five or more kinds of pharmaceuticals were ordered in $10 \%$ of cases, and ten or more units in $17 \%$. "Divided package sales" were more used for low-price drugs. These indicated that "divided package sales" seemed to be looked upon by pharmacies as a means of obtaining a small quantity of pharmaceuticals, and seemed to be used not only in an emergency but also routinely. There are several problems for wholesalers in operating "divided package sales", such as frequent delivery, delivery cost and information supply. Pharmacies should obtain a small quantity of pharmaceuticals by the routine delivery and should share the delivery cost. Pharmaceutical manufacturers' cooperation in printing necessary information on each immediate container will be useful. Though there could be alternative ways of obtaining a small quantity of pharmaceuticals, all of them have the matter of delivery. We believe "divided package sales" will contribute to this situation.
\end{abstract}

Key words__ divided package sales; small package pharmaceuticals; stock of pharmaceuticals; prescribing/dispensing split; delivery cost; shelf life

\section{INTRODUCTION}

A recent reform in Japan's health insurance system and a reduction in the price gap between market price and National Health Insurance (NHI) Drug Price has caused rapid progress in the prescribing /dispensing split and the number of pharmacies receiving prescriptions from more than one medical institution has increased. Since the NHI Price List includes more than 10,000 kinds of drugs, how pharmacies can stock many kinds of pharmaceuticals in their stores has become crucial, and obtaining small quantities is considered a key factor in addressing this. ${ }^{1)}$ The Ministry of Health and Welfare has defined small packages as in Table 1 and has asked pharmaceutical manufacturers to supply these. ${ }^{2)}$ According to an investigation by the Japan Pharmaceutical Association, however, pharmacies were not able to obtain small package pharmaceuticals by routine delivery in 1,555 cases (830 kinds of pharmaceuticals) between August 1997 and July 1998. ${ }^{3)}$ Moreover, the small packages supplied by the manufacturers were sometimes still too large for pharmacies to stock.

In these circumstances, Toho Yakuhin Co. Ltd., one of the leading wholesalers in Japan, in September 1998, began "divided package sales" in order to supply pharmacies with a small quantity. These "divided package sales" mean that wholesalers open an original package supplied by the manufacturer and sell only a part of the contents to fill an individual pharmacy's order.

Since "divided package sales" are one way of selling pharmaceuticals, there are several labeling re-

Table 1. Definition of Small Package Pharmaceuticals

\begin{tabular}{lc}
\hline \hline \multicolumn{1}{c}{ Dosage form } & Small package \\
\hline Tablets/Capsules & 100 tablets/capsules \\
Powders/Granules & $100 \mathrm{~g}$ \\
Syrups & $500 \mathrm{ml}$ \\
Injections & $10 \mathrm{ampules} /$ vials \\
Ointments/Creams & 10 tubes \\
Inhalants & 5 bottles \\
Eyedrops/Ear-drops/Nasal drops & 5 bottles \\
Cataplasms & $1 \mathrm{~kg}$ \\
Liquids & $100 \mathrm{ml}$ \\
Suppositories & $50 \mathrm{pieces}$ \\
Plasters & 50 pieces \\
\hline
\end{tabular}


quirements for goods under the Pharmaceutical Affairs Law, and such requirements are said to have obstructed these types of sales. In July 1992, a Ministerial Ordinance to the Pharmaceutical Affairs Law was amended to allow simplified labeling for goods sold in "divided package sales" by pharmacies or pharmaceutical association's supply centers. ${ }^{4)}$ In July 1999, this Ministerial Ordinance was further amended and the exemption from labeling requirements was expanded to "divided package sales" by wholesalers. ${ }^{5)}$

Toho Yakuhin set up a pharmacy handling only "divided package sales", which is called 'Pharmacy for Pharmacies'. This pharmacy supplies about 2,400 kinds of prescription drugs to pharmacies that are members of an 'ENIF Club' through “divided package sales". The reason Toho Yakuhin began handling these types of sales was that the exemption from labeling requirements had not yet been extended to wholesalers.

When a member pharmacy places an order for items, it uses a simple terminal called 'ENIF mini' to read bar-codes, and the order is automatically transmitted to the Pharmacy for Pharmacies through a telephone circuit. As a rule, items are delivered by regular delivery the next day. It is remarkable that Toho Yakuhin uses existing system for handling orders and delivery of items in "divided package sales". A related system necessary for these sales has also been developed by this company, which includes printing the production number and the shelf life on the items. The wholesale price of the pharmaceutical is the NHI Drug Price and the minimum ordering unit is one blister card of tablets, capsules or suppositories, or one tube or bottle of external drugs. One blister card usually contains ten or 14 tablets or capsules.

The purpose of this paper is to analyze "divided package sales" handled by Toho Yakuhin and to discuss how pharmacies should deal with the matter of stocking many kinds of pharmaceuticals in the future.

\section{RESULTS}

Figure 1 shows the number of orders Toho Yakuhin received and the sales amounts in "divided package sales" from July 1998, when Toho Yakuhin began the sales experiment, to December 1999. Both the number of orders and the sales amount have increased since the beginning of the service. The number of orders, 1,888 in September 1998, reached 23,000 in December 1999, and the sales amount, about 10 million-yen in September 1998, were 100 million-yen in December 1999. Figure 2 shows the number of pharmacies that have used "divided package sales": 32 at the beginning of the service and over 2,250 in December 1999.

We analyzed how many kinds of pharmaceuticals were included in each order during six months from July 1999 to December 1999. Figure 3 shows that one kind was included in $54 \%$ of all orders and only one or two kinds were ordered in $74 \%$ of all orders. Moreover, we see from Figure 4 how many units (the number of blister cards, tubes, or bottles) of pharmaceuticals were requested in each order: one unit in $20 \%$ of all orders and two units in $27 \%$. Thus, in about $50 \%$, the number of units was one or two.

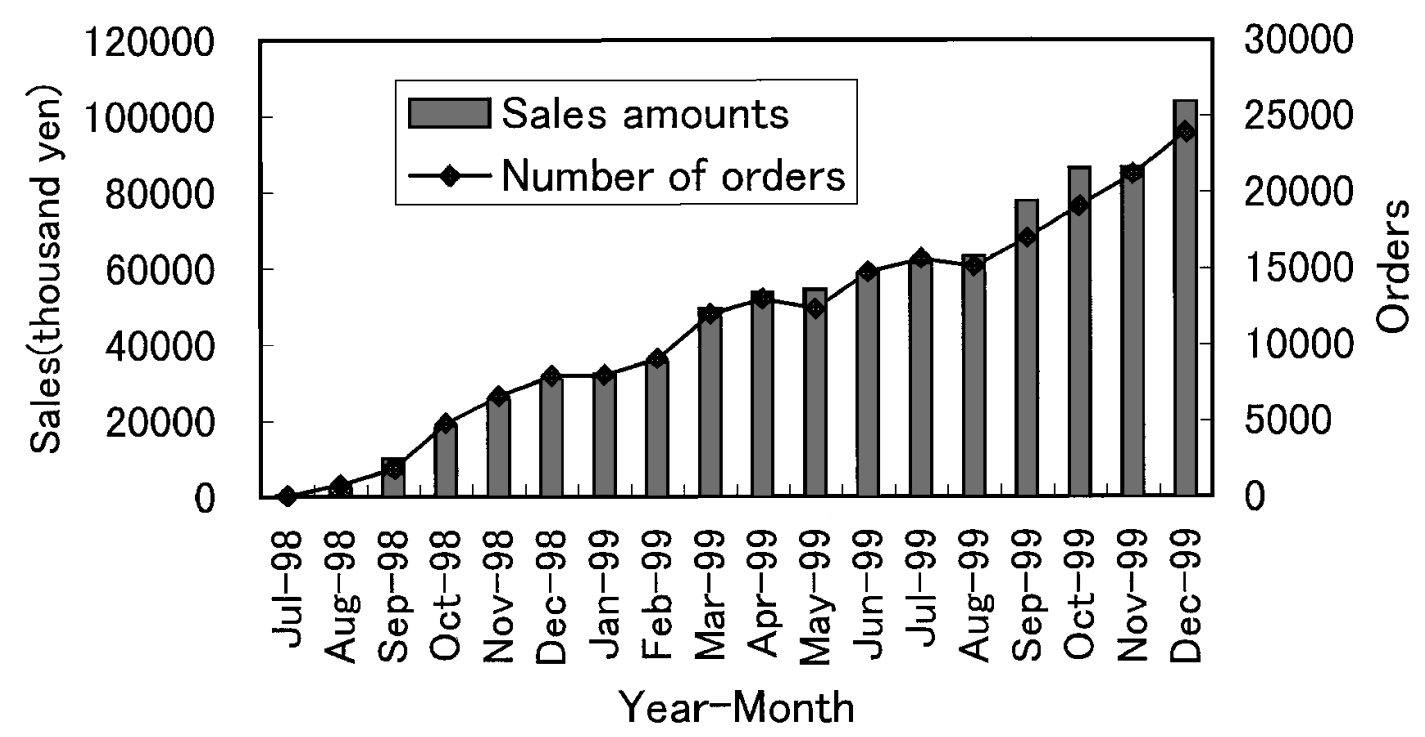

Fig. 1. Number of Orders and Sales Amounts in the Divided Package Sales 


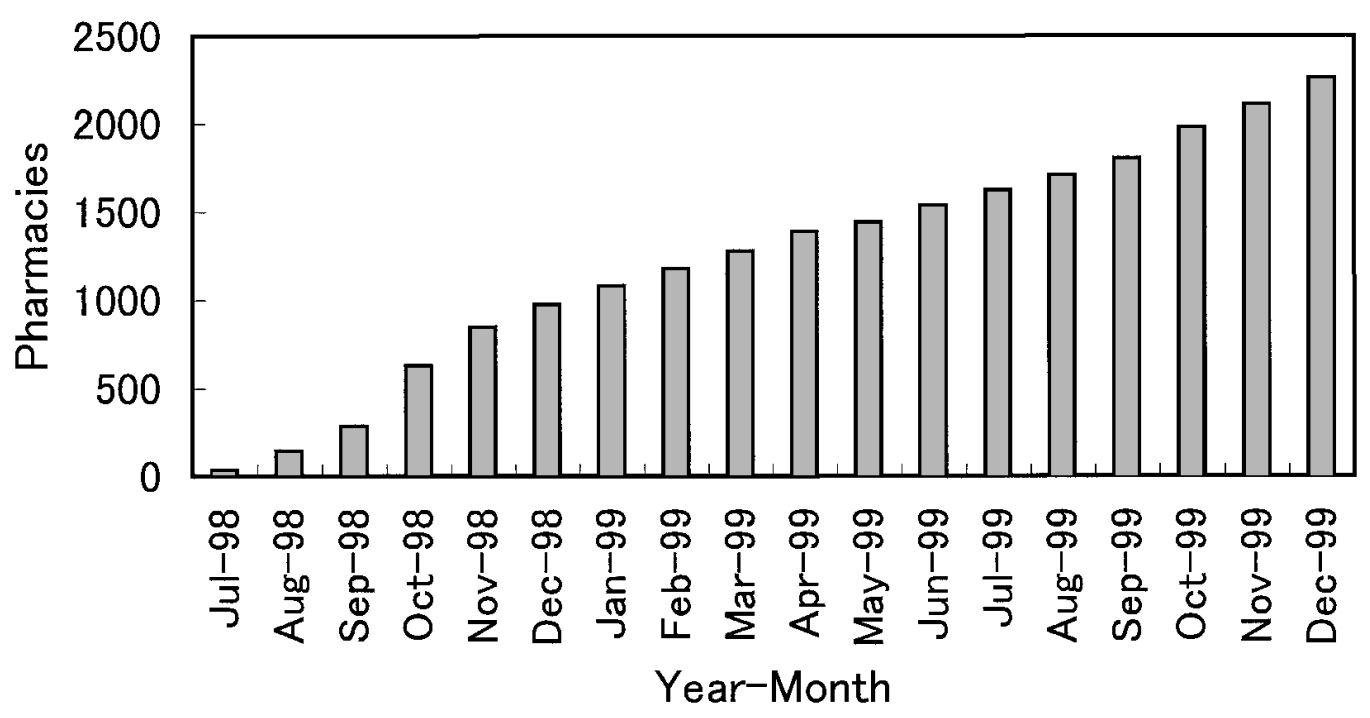

Fig. 2. Total Number of Pharmacies that Have Ordered

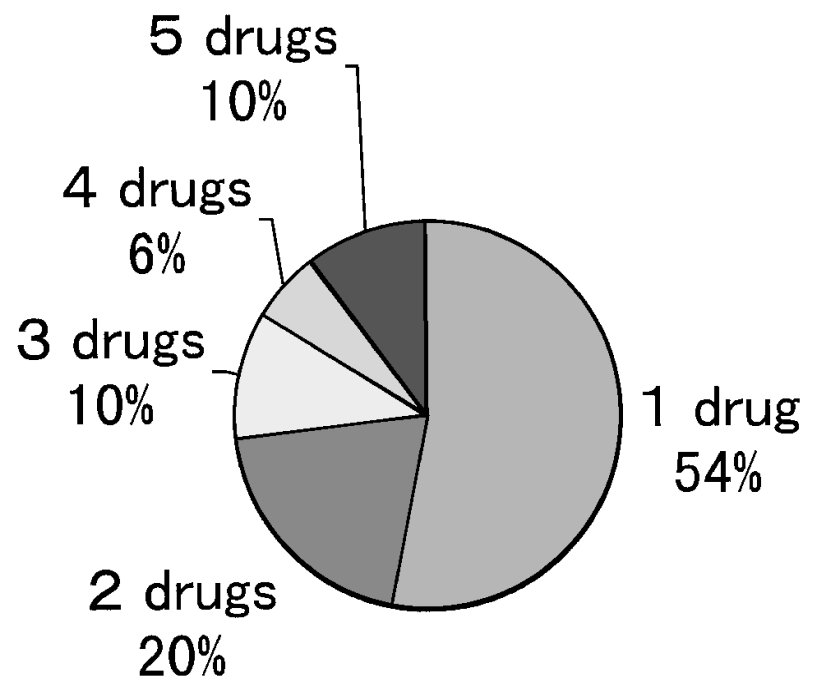

Fig. 3. Number of Kinds of Pharmaceuticals Included in Each Order

Next, we analyzed the relation between the number of orders and the NHI Drug Price. Figure 5 shows that most orders were made for drugs whose prices were 100 to 200 yen, and in $57 \%$ of all orders, the item price was less than 300 yen. Figure 6 shows the number of units of cheap pharmaceuticals ordered was the overwhelming majority and the prices were less than 300 yen in $77 \%$ of all units. Pharmacies seem to order more units than they need when they order a low-price drugs.

\section{DISCUSSION}

Being able to obtain a small quantity of pharmaceuticals is a key factor in pharmacies stocking

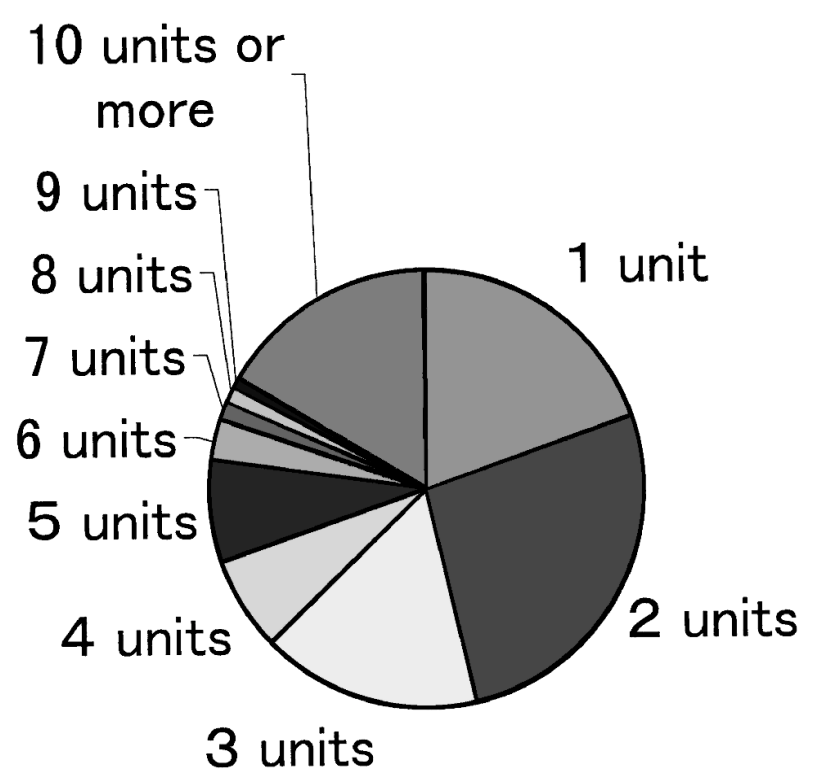

Fig. 4. Number of Units Ordered in Each Order

many kinds of pharmaceuticals, and "divided package sales" by wholesalers is seen as one means of making this possible. Figure 1 and Figure 2 show that the number of orders and the sales amount of "divided package sales", and the number of pharmacies using these sales have been on the increase.

It is assumed that pharmacies use "divided package sales" when they do not normally stock a drug and would have a large quantity of dead stock if they purchased a full package. One example is that the pharmacy may receive a prescription order for a drug to treat an acute disease. A second example is that a prescribed drug is a generic one, because pharmacies 


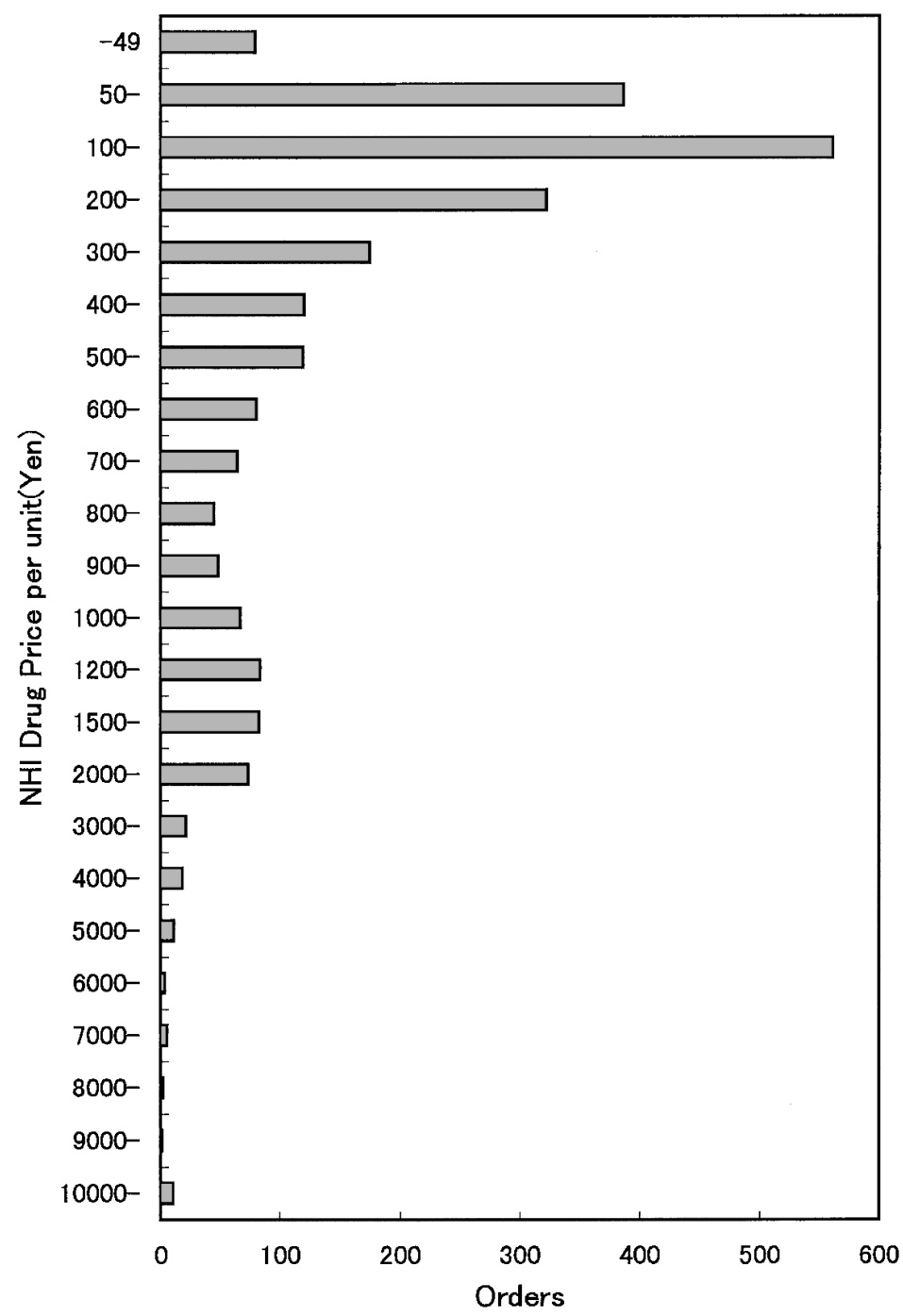

Fig. 5. Number of Orders in Relation to the NHI Drug Price

usually stock only one brand of a drug. In these two cases, pharmacies cannot necessarily anticipate that the drug included in the prescription will be prescribed again. However, it is necessary to note that Toho Yakuhin's “divided package sales" does not deal in generics. A third example is that the prescribed drug is a higher priced item, because a pharmacy suffers a great financial loss if the shelf life expires.

Figure 4 shows that one or two units were ordered in about $50 \%$ of cases. Since one unit of a pharmaceutical (ten or 14 tablets of capsules, three packages of one dose of granule, etc.) will be consumed at a time, these results imply that "divided package sales" are utilized to obtain drugs for which the pharmacy rarely receives a prescription order.

On the other hand, Figure 4 shows that ten or more units of pharmaceuticals were ordered in 17\% of cases. As ten or more units include more tablets or capsules than those needed for one prescription, it seems appropriate to assume that not a few pharmacies routinely use "divided package sales" to purchase these pharmaceuticals. Though we have no data analyzing the relation between the number of orders and the NHI Drug Price other than "divided package sales", Figures 5 and 6 show that these sales were used more for low-price drugs.

These results cause speculation, contrary to prior anticipation, that "divided package sales" have become a routine way of purchasing pharmaceuticals for most pharmacies. If pharmacies cannot expect a greater cost benefit from the price gap between purchasing price and the NHI Drug Price by purchasing pharmaceuticals in a large package, and if they cannot risk holding a large dead stock, it will be more profitable for them to use "divided package sales" frequently. Figure 7 shows the names of pharmaceu- 


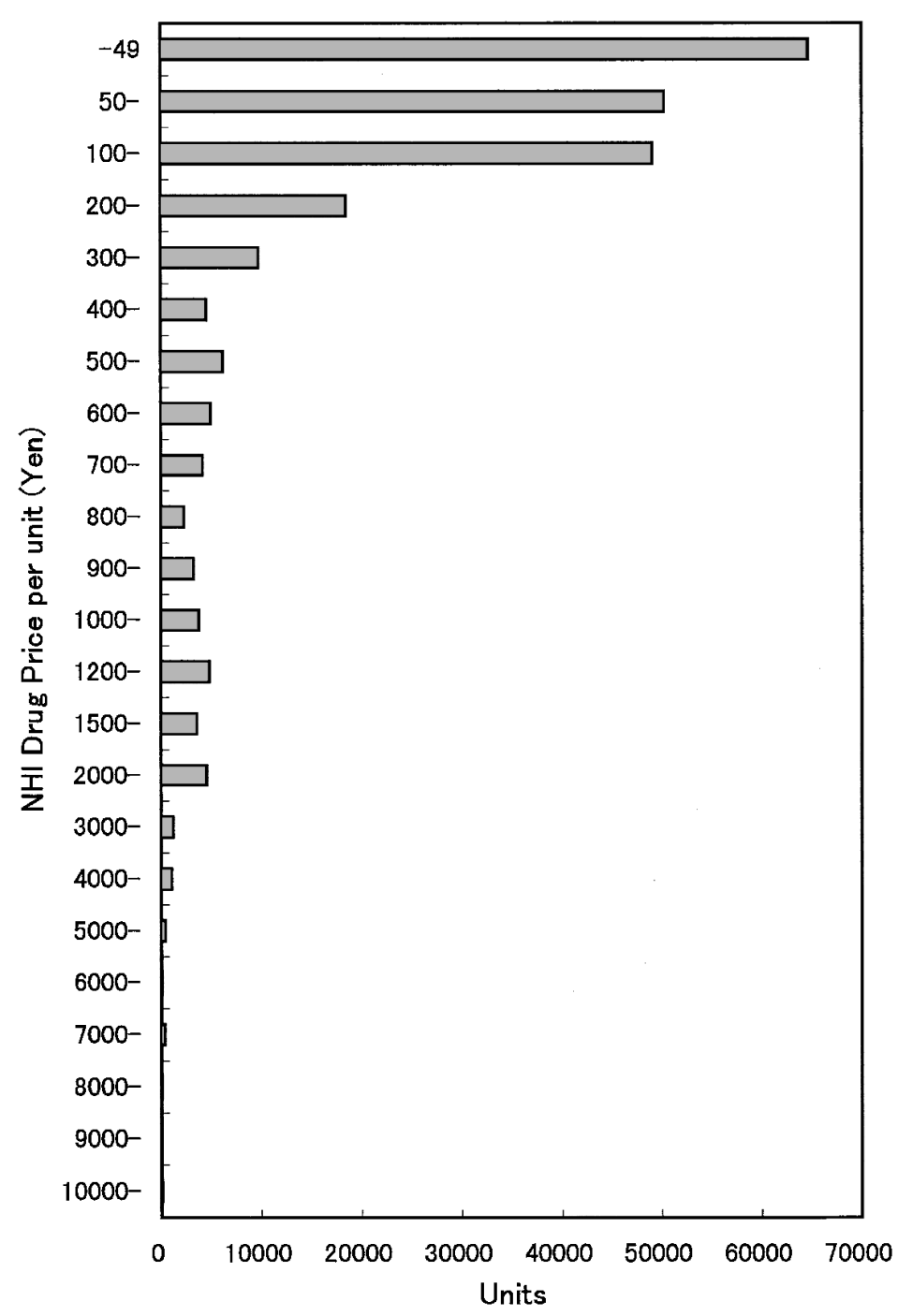

Fig. 6. Number of Units Ordered in Relation to NHI Drug Price

ticals by number of units ordered through "divided package sales". Toho Yakuhin sold about 6,800 kinds of pharmaceuticals other than injections in April 2000 through all its sales channels including "divided package sales". If we put those products in order of sales quantity and compare with the result shown in Figure 7, nine products among the pharmaceuticals listed in this figure were included in the first 100 in the sales quantity in the company's overall sales. Thus pharmaceuticals listed in the figure are believed to be frequently dispensed, suggesting that our speculation is valid.

Besides "divided package sales" by wholesalers, there could be alternative ways of obtaining a small quantity of pharmaceuticals. Pharmacies may obtain them from manufacturers or a pharmaceutical association's supply center, for example. They may also obtain them from other pharmacies by exchanging information on their inventories. The optimal way will depends on how the prescribing/dispensing split in the community progresses. The number, scale or distribution of medical facilities that are issuing prescriptions, the distribution of pharmacies and patients' behavior should be considered.

Whichever way is decided, however, there is the matter of delivery. For example, though pharmacies may be able to learn that some supply center or pharmacy has the drug, who should go there to get it? Manufacturers may be able to supply a small package of pharmaceuticals on demand, but it is not enough to maintain a small, stable supply. It seems reasonable to suppose that this will lead pharmacies to order a small quantity every time. Consequently, wholesalers will be asked to provide the same service as that in “divided package sales". Since small packages still contain a large amount of pharmaceuticals for pharmacies in some cases, the supply of such small package of pharmaceuticals by manufacturers still needs 


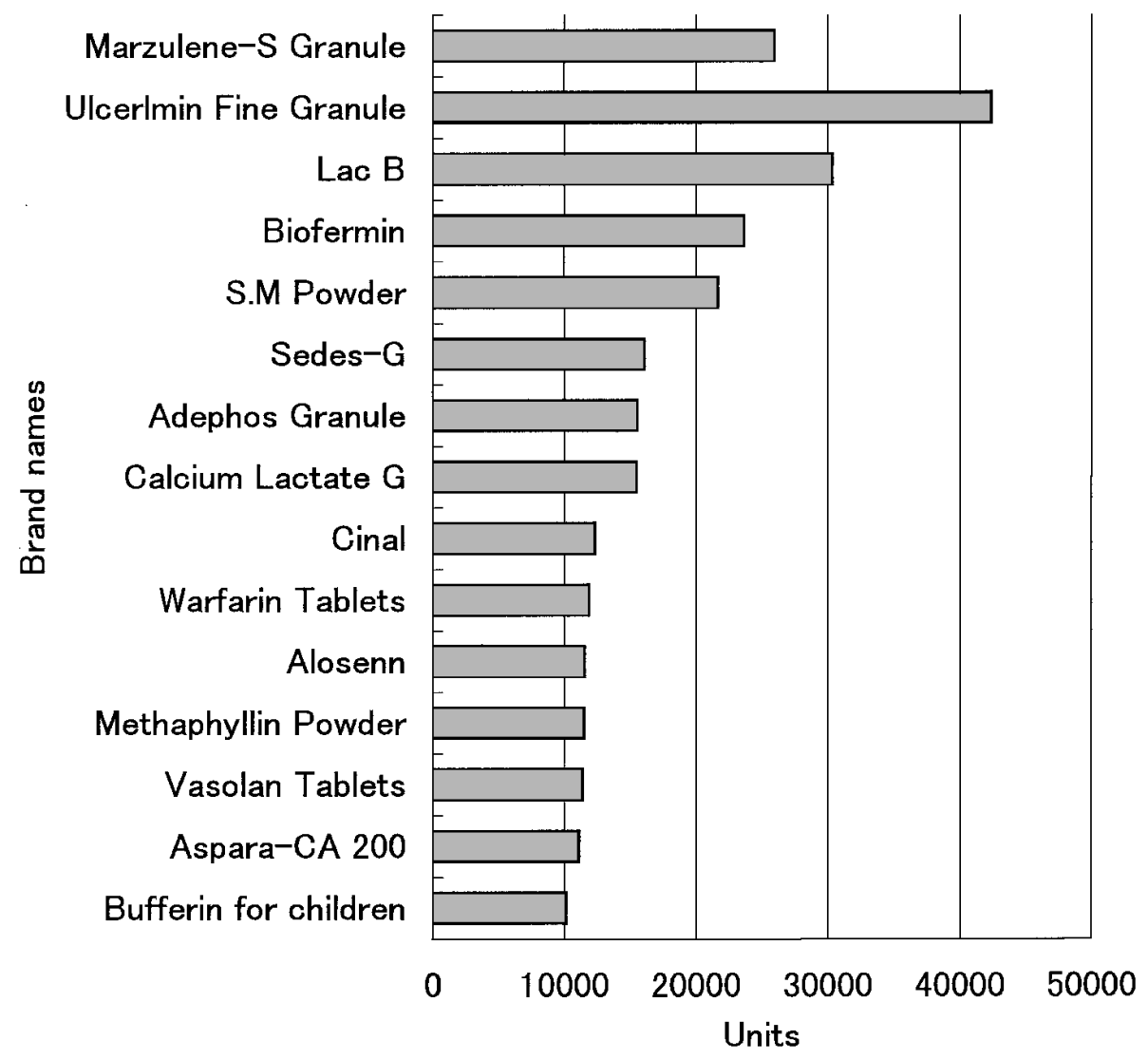

Fig. 7. Number of Pharmaceuticals for which Many Units were Ordered

to be complemented by "divided package sales" by wholesalers.

Thus the necessity for "divided package sales" by wholesalers will increase, but there are several problems in wholesalers assuming the responsibility for supplying a small quantity of pharmaceuticals by this means. First of all, if pharmacies purchase pharmaceuticals little by little in spite of great demand, this will necessitate frequent deliveries. As reported above, the tendency for pharmacies to purchase in small quantities has already been observed. In addition, it is likely that the more pharmacies purchase in small quantities, the more they will request urgent delivery of wholesalers so as not to keep a patient waiting.

The second point we should raise is the matter of delivery cost. Delivery cost does not depend on the price of the pharmaceuticals delivered. If "divided package sales" are used by pharmacies to purchase cheaper drugs, their returns cannot cover the delivery cost. Wholesalers will be forced to ask users to share some of the cost.

The third point is the matter of information supply. When a wholesaler divides a package of pharmaceuticals into several segment and delivers them to various pharmacies, the manufacturers and the wholesalers need to make it clear how they would share the responsibility for information supply. ${ }^{5)} \mathrm{Un}$ less the wholesaler can trace the pharmaceuticals they have sold, there is some fear of becoming lax in measures to protect consumers' health.

We would like to suggest that, by purchasing a small quantity of pharmaceuticals, pharmacies should not reduce the cost necessary to stock them but should increase the kinds they are stocking. If they have one blister card of a pharmaceutical in stock, they can dispense it now and will be able to dispense the rest of the prescription order later. In so doing, a pharmacy will not cause inconvenience to a patient and will fulfill its obligation. This makes it possible to maintain a regular delivery, to keep delivery cost down and to provide a stable service of "divided package sales". Urgent deliveries will not only raise delivery cost up but also disturb the routine delivery system.

On the other hand, pharmaceutical manufacturers are requested to cooperate with wholesalers for “divided package sales". For example, if manufacturers can print the information required by law such as the shelf life on each immediate container, it would 
be very useful from the viewpoint of safety and efficiency.

\section{CONCLUSION}

Toho Yakuhin's year-long experience in "divided package sales" has shown that these have been utilized to obtain drugs for which a pharmacy rarely receives a prescription order. At the same time, not a few pharmacies have routinely used "divided package sales" for purchasing pharmaceuticals. Thus these sales seem to be looked upon by pharmacies as a means of obtaining a small quantity of pharmaceuticals. The experience has also shown there are several problems for wholesalers in operating "divided package sales", such as frequent delivery, delivery cost and information supply. Joint effort by pharmacies and manufacturers in providing "divided package sales" is expected.

There is an opinion that the matter of stocks of pharmaceuticals arises only at the beginning of the prescribing/dispensing split and at its expanding stage. ${ }^{6}$ If so, the matter will vanish when the prescribing/dispensing split takes root. However, since pharmacies will increasingly purchase pharmaceuticals in a small quantity if possible, building a system providing such a service is urgent. The necessity of "divided package sales" by wholesalers is expected to continue to increase.

The Prescribing/dispensing split is expected to progress and it seems fair to say that whether pharmacies are able to solve the matter of stocking phar- maceuticals or not may decide its future. We believe "divided package sales" will contribute to this situation, and therefore hope that all parties concerned pharmacies, wholesalers and manufacturers - will earnestly consider this matter.

\section{REFERENCES}

1) Japan's Ministry of Health and Welfare, "Study group's report: Manual for reserving and controlling pharmaceuticals in a pharmacy," March 1999.

2) Notice of the Director General of Pharmaceutical Affairs Bureau, MHW, notice No. 293, "Smooth supply of small package pharmaceuticals." 27 March 1992.

3) Japan Pharmaceutical Association, "The results of a survey through questionnaires on small package pharmaceuticals particularly for generics," 25 Feb. 1999.

4) Notice of the Director General of Pharmaceutical Affairs Bureau, MHW, notice No. 418, "Amendment of Ministerial Order to Pharmaceutical Affairs Law and Narcotics and Psychotropics Control Law," 1 May 1992.

5) Notice of the Director General of Pharmaceutical Affairs Bureau, MHW, notice No. 905, "Amendment of Ministerial Order to Pharmaceutical Affairs. Law,” 28 July 1999.

6) Program of the 32nd Japan Pharmaceutical Association Congress of Pharmacy and Pharmaceutical Science, 1999, pp. 112-115. 